Original Research Paper

\title{
Analysis of Non-Equilibrium Phase Change in Transfers at Low Water Content by Considering the Film Flow
}

\author{
${ }^{*}$ Marcel Bawindsom Kébré, ${ }^{2}$ François Ouédraogo, \\ ${ }^{2}$ Bétaboalé Naon, ${ }^{3}$ Fabien Cherblanc and ${ }^{1}$ François Zougmoré \\ ${ }^{I}$ Département de Physique, Université Joseph KI-ZERBO, Ouagadougou, Burkina Faso \\ ${ }^{2}$ Département de Physique, Université Nazi BONI, Bobo Dioulasso, Burkina Faso \\ ${ }^{3}$ Laboratoire de Mécanique et Génie Civil (LMGC), Université de Montpellier, CNRS, Montpellier, France
}

Article history

Received: $30-12-2020$

Revised: 27-03-2021

Accepted: 01-04-2021

Corresponding Author: Marcel Bawindsom Kébré Département de Physique, Université Joseph KI-ZERBO, Ouagadougou, Burkina Faso Email: mkebre@gmail.com

\begin{abstract}
In this study, we analyze the effect of non-equilibrium phase change on transfer at low water content in a sandy soil by considering the contribution of film flow in the motion of liquid water. Indeed, most of the non-equilibrium study methods use for hydraulic conductivity, the van Genuchten-Mualem (VGM) capillary model which does not consider the film flow occurred at low water contents. Thus, we conduct a theoretical study by using an unidirectional non-equilibrium two phase flow model to simulate water transfers by filtration of liquid water and diffusion of water vapour coupled by liquid/gas phase change. We then compare the results of the non-equilibrium with the classical model of VGM for hydraulic conductivity function and another model considering the film flows. The numerical simulation is based on a column of sandy soil exposed to a controlled atmosphere. We observe that the shapes of the profiles of fluxes (liquid water, liquid/vapour non-equilibrium phase change) simulated using the capillary model are very different from those obtained with the model considering the film flow. In this last case, the liquid/gas nonequilibrium is not noticeable as in the case of the capillary model. It seems that the film flows occult the water vapour diffusion by delaying the phase change process, therefore a lower concentration of water vapour into the soil than in the case where the capillary flow alone has been considered.
\end{abstract}

Keywords: Sandy Soil, Low Water Content, Phase Change, NonEquilibrium, Film Flow, Hydraulic Conductivity

\section{Introduction}

The phase change (evaporation and condensation) is the central phenomenon which governs exchanges at the ground-atmosphere interface (Smits et al., 2011). The majority of the water is liquid form in the soil must pass into vapour form to migrate to the atmosphere. In the funicular regime (high water contents), it is generally accepted that the process phase change takes place on the ground surface (Mitarai and Nori, 2006). However, when the content in water drops and the medium enters the pendulum then hygroscopic domain, the localization of evaporation remains an open problem (Levitt and Young, 2007). Previous results locate the phase change process in the first few inches from the ground (Chammari et al., 2008; Ouedraogo et al., 2013). This phase change allows to describe the nonequilibrium state in a porous media which occur at the dry end i.e., at low water content. Thus, the modeling of water dynamics in this moisture state of the soil deserves special attention.

As water content decreases, hygroscopic effects become predominant and adsorptive forces prevail over capillary ones. Liquid water is present in the form of adsorbed layers around soil particles and its thermodynamic state can differ markedly from bulk water (Kébré et al., 2017). Therefore, the modelling of transport phenomena at low water contents requires a specific description (Kébré et al., 2017). In particular, it has been emphasized that vapour diffusion can contribute considerably to water transport, which leads to reconsidering the classical assumption of a local equilibrium (Smits et al., 2011; Ouedraogo et al., 2013; Massman, 2015). In such situations, liquid filtration, vapour diffusion and phase change are simultaneous 
transport processes that must be considered to describe global water exchanges accurately in natural soil.

Moreover, the physical processes that govern liquid water flow in the low water content range are still in question. In general, numerical modelling based on the Richards equation requires the complete determination of its hydraulic properties (i.e., the hydraulic conductivity and water retention functions) (Kébré et al., 2017). Numerous attempts have been made to derive theoretically the hydraulic conductivity function directly from the water retention curve based mainly on capillary bundle models (Burdine, 1953; Mualem, 1976). Although fairly accurate at large water contents where water is held mainly by capillary forces, these descriptions generally underestimate the permeability at low water contents (Khaleel et al., 1995). The most used van Genuchten-Mualem permeability function is appropriate for intermediate water contents $\left(w>0.04 \mathrm{~kg} \cdot \mathrm{kg}^{-1}\right)$, which highlights the shortcomings of capillary bundle models in predicting the permeability function in this dry range of the soil (Kébré et al., 2017).

During the past decade, several authors have emphasized the major contribution of flow in thin liquid films, which is likely to become important at low water contents. To address this issue, in (Kébré et al., 2017), we have made discussions on water flow at low water content and the contributions of film flow to more predict water profiles for sandy soils. A relative permeability function has been proposed to successfully describe water flow focusing on low water content.

Another difficulty to describe drying or water management processes specially at low water content is the formulation of the constitutive equations of the water (vapour and liquid) transport models. Most of them relies on the local equilibrium assumption (Ouedraogo et al., 2013). This hypothesis specifies that, on each point of the domain, liquid and gas phases are in equilibrium (Li et al., 2019; Seredyński et al., 2020). In that case, it means that the vapour pressure remains at its equilibrium value (Ouedraogo et al., 2013). Physically, it amounts to saying that, when compared to diffusion, phase change processes are sufficiently fast to assume it instantaneous. This usual assumption has been considered without carefully checking its domain of validity (Ouedraogo et al., 2013; Seredyński et al., 2020). In the fully equilibrium model, changes in the moisture content of the liquid and vapor forms are established immediately, so water content in the liquid and vapor phases are tightly coupled by a global term (Seredyński et al., 2020). This situation then leads to a single equation to describe the movement of water in the porous medium.

Therefor, a large set of experimental investigations on phase change kinetics have suggested that evaporation in soils may not be as fast as supposed (Chammari et al., 2008; Lozano et al., 2008). For instance, phase change kinetics is drastically decreased when the binding energy of water layers adsorbed on finescale grains increased. This is mainly the case in the hygroscopic range of water content and is emphasized in the presence of a clayey fraction (Ouedraogo et al., 2013).

That is why, more recently, several authors have discussed this equilibrium state in transfers within porous media in the full range of water contents in the soil (Smits et al., 2011; Halder et al., 2011; Ouedraogo et al., 2013; Nuske et al., 2014; Massman, 2015; Trautz et al., 2015; Novak, 2019; Li et al., 2019). In particular, (Ouedraogo et al., 2013) proved that this liquid-gas thermodynamic equilibrium during transfers in the soil is called into question on the basis of a series of experiments relating to soil at low water content.

However, most of these studies did not consider the contribution of film flow in the soil at the dry end. They commonly used the (Van Genuchten, 1980; Mualem, 1976) capillary model for the hydraulic conductivity function which does not consider the film flow occurred at low water contents thus neglecting the contribution of the transport of liquid water in film form within the porous matrix. While, we had previously shown the existence of this mode of transport (Kébré et al., 2017), in this study, we discuss the non-equilibrium phase change effect on the transfer in the sandy soil when considering the film flows. We focus on nonequilibrium model knowing that many works are make comparison between equilibrium and non-equilibrium in the water dynamic in the porous media (Smits et al., (2011; Li et al., 2019; Seredyński et al., 2020; Tamizdoust and Ghasemi-Fare, 2020)

\section{Materials and Methods}

\section{Basic Soil Properties}

\section{Textural and Physical Properties}

The upper layer of a natural Arenosol (IUSS Working Group WRB, 2006) from Nasso, Burkina Faso (latitude $11.210733^{\circ}$, longitude $-4.420386^{\circ}$ ), were investigated. The soil sample, denoted $\mathrm{NH} 1$, was taken from the top layer of the soil (0-30-cm depth). According to the International Society of Soil Science (ISSS) classification, the textures of these samples are sand. The main morphological properties of the soil sample are summarized in Table 1.

Table 1: Textural characteristics, dry density $\left(\rho_{s}\right)$, porosity $(n)$, saturated gravimetric water content $\left(w_{s a t}\right)$ and saturated conductivity $\left(K_{\text {sat }}\right)$

\begin{tabular}{lllllll}
\hline & $\begin{array}{l}\text { Silt } \\
\text { Sand }\end{array}$ & Clay & $\begin{array}{l}\rho_{s} \\
\mathrm{~kg} . \mathrm{m}_{3}{ }^{-}\end{array}$ & $\begin{array}{l}\mathrm{n} \\
-\end{array}$ & $\begin{array}{l}w_{\text {sat }} \\
\mathrm{kg} . \mathrm{kg}_{1}{ }^{-}\end{array}$ & $\begin{array}{l}K_{\text {sat }} \\
\mathrm{m} . \mathrm{s}^{-1}\end{array}$ \\
\hline 90 & 7 & 3 & 1688 & 0.36 & 0.215 & $3.65 \times 10^{-5}$ \\
\hline
\end{tabular}




\section{Water Activity}

The liquid activity is defined by the sorption isotherm curve. Since it is an equilibrium property, it can only describe an equilibrium situation as required by the local equilibrium assumption (Aljerf, 2018). Rejecting this fundamental hypothesis means that the vapour pressure, $p_{v}$, can diverge from its equilibrium value, $p_{v e q}$. For the case of an unsaturated soil, we were inspired by the model representing the relationship between the chemical potential and the water content proposed by (Fredlund and Xing, 1994) to model the isotherms. Thus, the water activity in the soil is given by:

$a_{w}=\frac{p_{v e q}(T, w)}{p_{v s}(T)}=1-\left[\ln \left(e+\left(\frac{w}{A}\right)^{B}\right)\right]^{C}$

where, $A, B$ and $C$ are empirical parameters, the values of which for the soil under study are recorded in Table 2. Figure 1 represents the activity of water measured experimentally using the salt solutions method compared with the modeled one.

\section{Soil Hydraulic Conductivity Functions}

The soil water retention curve and the relative hydraulic conductivity of this sandy soil have been presented in (Kébré et al., 2013; 2017). The models used in this study are given below:
- Van Genuchten (1980) model (denoted by VG80) for the soil water retention curve expressed with water chemical potential $\mu\left(\mathrm{J}_{\mathrm{kg}} \mathrm{kg}^{-1}\right)$ :

$$
S_{e}=\frac{w-w_{r}}{w_{s a t}-w_{r}}=\left[\frac{1}{1+(\alpha \mu)_{n}}\right]^{m}
$$

- Van Genuchten-Mualem model (denoted by VG80-M) for the relative hydraulic conductivity without film flows:

$$
K_{r}\left(S_{e}\right)=\sqrt{S_{e}}\left[1-\left(1-S_{e}^{\frac{1}{m}}\right)^{m}\right]^{2}
$$

- The relative hydraulic conductivity with film flows from (Kébré et al., 2017) (denoted by K17):

$$
K_{r}\left(S_{e}\right)=K_{r}^{c a p}+K_{r}^{f i l m}=\sqrt{S_{e}}\left[1-\left(1-S_{e}^{\frac{1}{m}}\right)^{m}\right]^{2}+\beta S_{e}^{\gamma}
$$

Table 2: Coefficients of Equation (1) with the statistics $\mathrm{R}^{2}$

\begin{tabular}{|c|c|c|c|c|}
\hline \multicolumn{3}{|c|}{ Parameters of the model } & \multicolumn{2}{|c|}{ Statistics } \\
\hline A & B & $\mathrm{C}$ & $\mathrm{R}^{2}$ & RMSE \\
\hline kg. $\mathrm{kg}^{-1}$ & - & - & $\%$ & kg.kg ${ }^{-1}$ \\
\hline 0.001935 & 5.144 & -1.061 & 99.01 & 0.03677 \\
\hline
\end{tabular}
and Root Mean Square Error (RMSE) for the accuracy of prediction

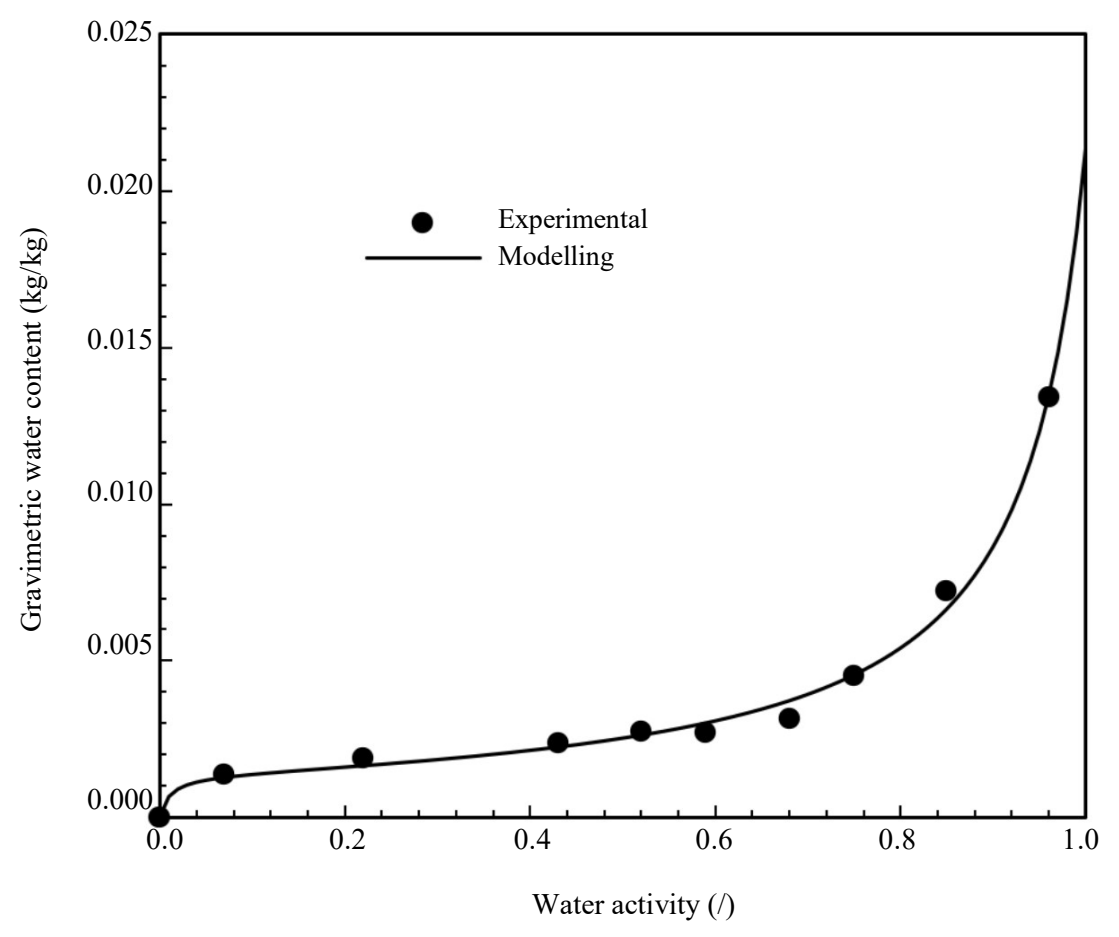

Fig. 1: Water activity for NH1 
The first term of this equation corresponds to the relative permeability coefficient according to the VG80 $\mathrm{M}$ model for high and intermediate water content values and the second term, a simple expression to take account the film flow at low water content. This formulation give good results in inverse analysis for sandy soil liquid water transfer at low water content (Kébré et al., 2017).

In these equations, $S_{e}$ is the effective saturation, $w_{r}$ $\left(\mathrm{kg} . \mathrm{kg}^{-1}\right)$ and $w_{\text {sat }}\left(\mathrm{kg} . \mathrm{kg}^{-1}\right)$ are the residual and saturated water content, respectively, $n, m, \beta$ and $\gamma$ are fitting parameters.

Table 3 give values of coefficients for Eq. (2-4) for the water retention curve and Table 4 for the relative hydraulic conductivity for Eq. (4). The statistics $\mathrm{R}^{2}$ and Root Mean Square Error (RMSE) for the accuracy of prediction are shown.

Figure 2 gives a representation of the water retention curve of the soil under study while Figure 3 compares the relative permeability on the one hand without considering the film flow (Eq. 3) and on the other hand with the film flow (Eq. 4). It is clearly noted that not taking the film flow underestimates the transfer force including the evolution of water content in the soil (Kébré et al., 2017).

\section{Computational Approach}

\section{Conservation Equations}

Three elementary phenomena must be considered in the simulation of the transfers of liquid water and its vapour: Filtration of the liquid phase induced by capillary and gravity forces; the diffusion of vapour in the gas phase and liquid-vapour water phase change. The mass balance equations for each phase of the water component are then:

- Liquid water:

$$
\frac{\partial \rho_{w}}{\partial t}+\nabla \cdot\left(\rho_{w} v_{w}\right)=-\hat{\rho}
$$

- Water vapour:

$$
\frac{\partial \rho_{v}}{\partial t}+\nabla \cdot\left(J_{v}\right)=+\hat{\rho}
$$

Table 3: Coefficients for the water retention curve model (Eq. 2) (Kébré et al., 2017)

\begin{tabular}{llllll}
\hline Parameters of model & & & \multicolumn{2}{l}{ Statistics } \\
$-w_{r}$ & $\alpha$ & $n$ & $m$ & $\mathrm{R}^{2}$ & $\mathrm{RMSE}$ \\
$\mathrm{kg} \mathrm{kg}^{-1}$ & $\mathrm{~J} \mathrm{~kg}^{-1}$ & - & - & $\%$ & $\mathrm{~kg} \mathrm{~kg}^{-1}$ \\
\hline 0 & 0.912 & 1.392 & $1-1 / \mathrm{n}$ & 99.30 & 0.0041 \\
\hline
\end{tabular}

Table 4: Additional coefficients for the relative hydraulic

\begin{tabular}{|c|c|c|c|}
\hline \multicolumn{2}{|c|}{ Parameters of model } & \multicolumn{2}{|c|}{ Statistics } \\
\hline$\beta$ & $\gamma$ & $\mathrm{R}^{2}$ & RMSE \\
\hline- & - & $\%$ & $\mathrm{~kg} \mathrm{~kg}^{-1}$ \\
\hline $2.405 \times 10^{-6}$ & 1.981 & 97.84 & 0.002141 \\
\hline
\end{tabular}
conductivity (Kébré et al., 2017)

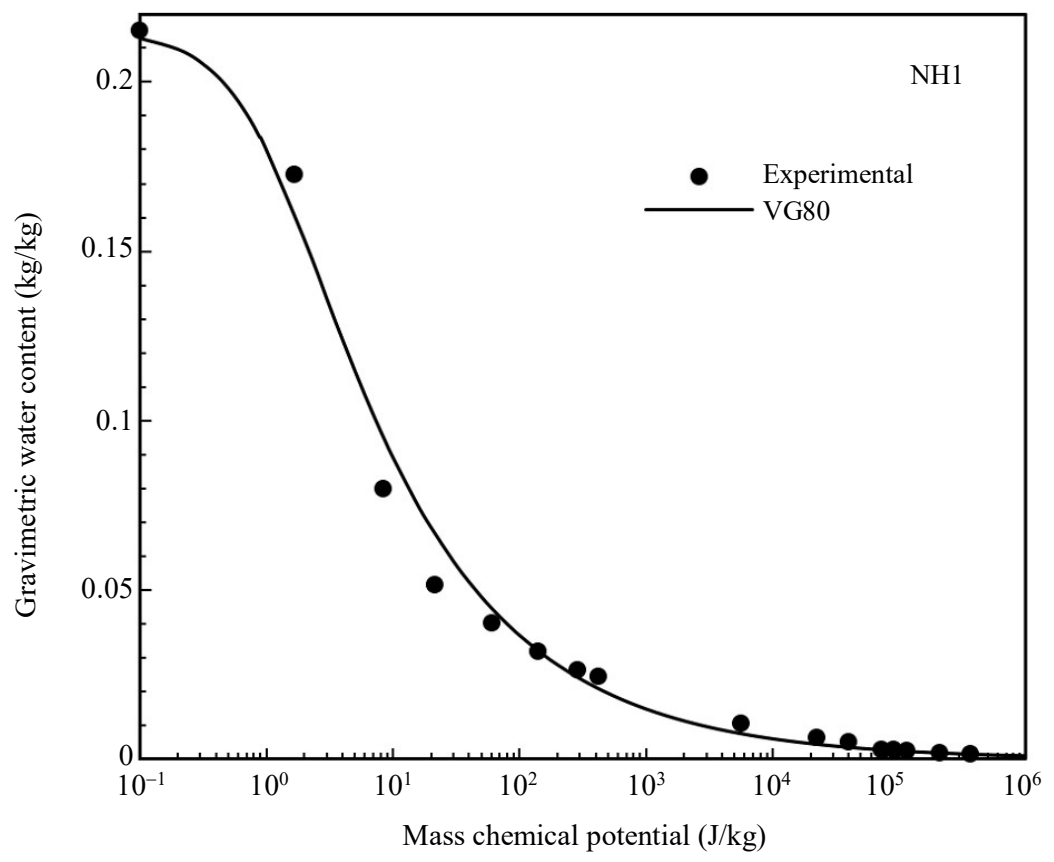

Fig. 2: Water retention curve for NH1 with Van Genuchten (1980) model (VG80) 


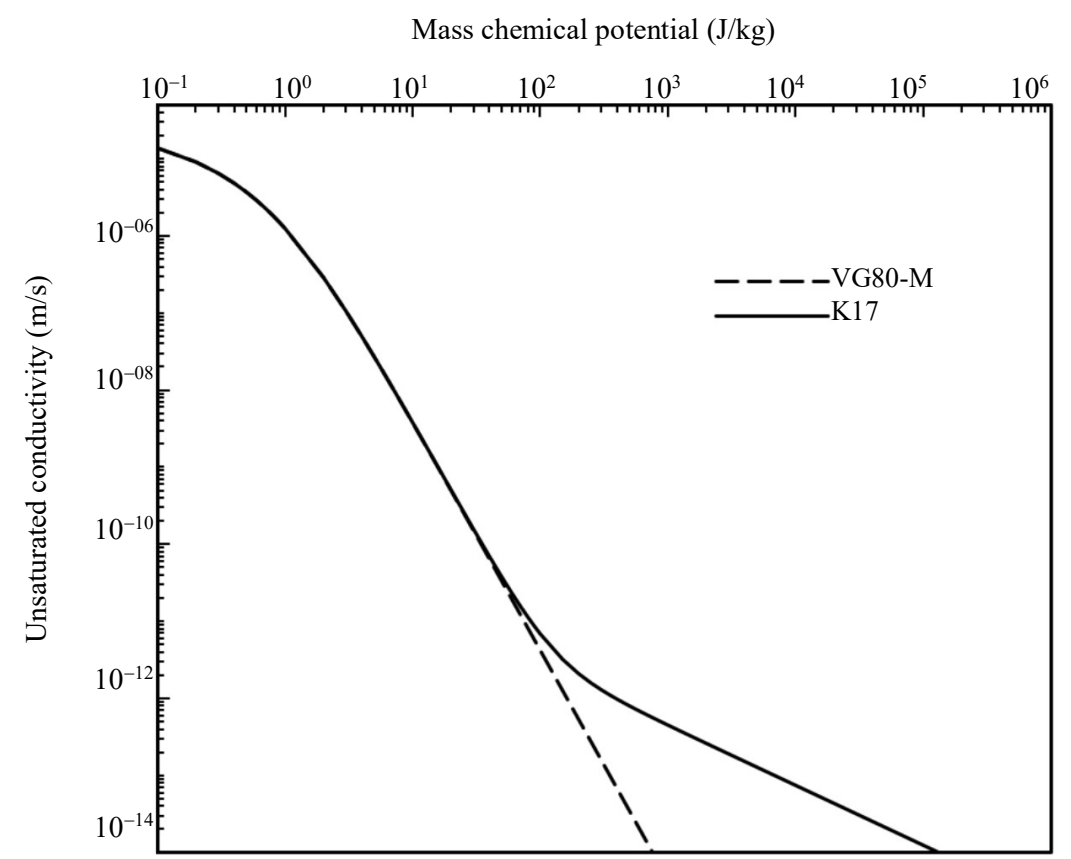

Fig. 3: Unsaturated hydraulic conductivities as a function of the chemical potential for NH1 soil: a comparison between the results from VG80-M and the model K17 considering the film flows plotted on a logarithmic scale

In these relations (Eq. 5 and 6), $\rho_{w}$ and $\rho_{v}\left[\mathrm{~kg} \cdot \mathrm{m}^{-3}\right]$ denote the bulk densities of liquid water and water vapour respectively; $v_{w}\left[\mathrm{~m} \cdot \mathrm{s}^{-1}\right]$ is the intrinsic velocity of liquid water and $J_{v}\left[\mathrm{~kg} \cdot \mathrm{m}^{-2} \cdot \mathrm{s}^{-1}\right]$ is the vapour diffusion flux. The rate of the liquid-vapour phase change $\hat{\rho}\left[\mathrm{kg} \cdot \mathrm{m}^{-3} \cdot \mathrm{s}^{-1}\right]$ is thus a voluminal scalar flow, representing the mass of water passing from the liquid state to the vapour state per unit of volume and unit of time.

The model state variables, defined from an experimental point of view, are:

- The gravimetric water content, $w\left[\mathrm{~kg}^{\mathrm{kg}} \mathrm{kg}^{-1}\right]$, the ratio between the bulk densities of water liquid and solid particles

- The partial pressure of water vapour in the gas phase, $p_{v}[\mathrm{~Pa}]$, related to the apparent density apparent of water vapour $\rho_{v}$ through ideal gas law:

$$
\phi_{g} p_{v}=\frac{R T}{M_{w}} \rho_{v}
$$

where, $\phi_{g}[-]$ is the volume fraction occupied by the gas phase; $T[\mathrm{~K}]$, the temperature; $R\left[\mathrm{~J} \cdot \mathrm{mol}^{-1} \cdot \mathrm{K}^{-1}\right]$ the ideal gas constant and $M w\left[\mathrm{~kg} \cdot \mathrm{mol}^{-1}\right]$, the molecular molar mass of water.

By explaining the liquid water and vapour fluxes, the conservation equations are in the form below (Kébré, 2013):

$$
\left\{\begin{array}{l}
\frac{\partial w}{\partial t}-K_{s a t} \frac{\rho_{w}}{\rho_{s} g} \nabla \cdot\left(K_{r} \frac{\partial \mu}{\partial w} \nabla w-K_{r} g\right)-\frac{\hat{\rho}}{\rho_{s}}=0 \\
\frac{\partial}{\partial t}\left(\phi_{g} p_{v}\right)-\nabla \cdot\left(D_{v s} \nabla p_{v}\right)+\frac{R T}{M_{w}} \hat{\rho}=0
\end{array}\right.
$$

$D_{v s}\left[\mathrm{~m} . \mathrm{s}^{-2}\right]$ is the diffusion coefficient of the gas in the porous medium which depends on several parameters including the gas diffusion coefficient in free air $D_{v a}$ $\left[\mathrm{m} . \mathrm{s}^{-2}\right]$, the path traveled represented by the tortuosity factor $\tau[-]$ and the volume fraction of the gas phase $\phi_{g}$. The following expression from (Moldrup et al., 2000) (Eq. 9) is the most used with (Millington and Quirk, 1961) model for the tortuosity factor and $D_{v a}=26.110^{-6}$ $\mathrm{m} . \mathrm{s}^{-2}$ from (Campbell, 1985):

$D_{v s}=D_{v a} \phi_{g} \tau=D_{v a} \phi_{g} \frac{\phi_{g}^{7 / 3}}{n^{2}}$

\section{Liquid-Gas Phase Change}

In isothermal case, the rate of non-equilibrium phase change (Eq. 10) is expressed as a function of the partial vapour pressure $\left(p_{v}\right)$; the equilibrium vapour pressure $(\mathrm{Eq}$. 1) and a phenomenological coefficient, $L$ [kg.K.s.m ${ }^{-5}$, determined experimentally (Lozano et al., 2008):

$\hat{\rho}=-L \frac{R}{M_{w}} \ln \left(\frac{p_{v}}{p_{\text {veq }}}\right)$ 


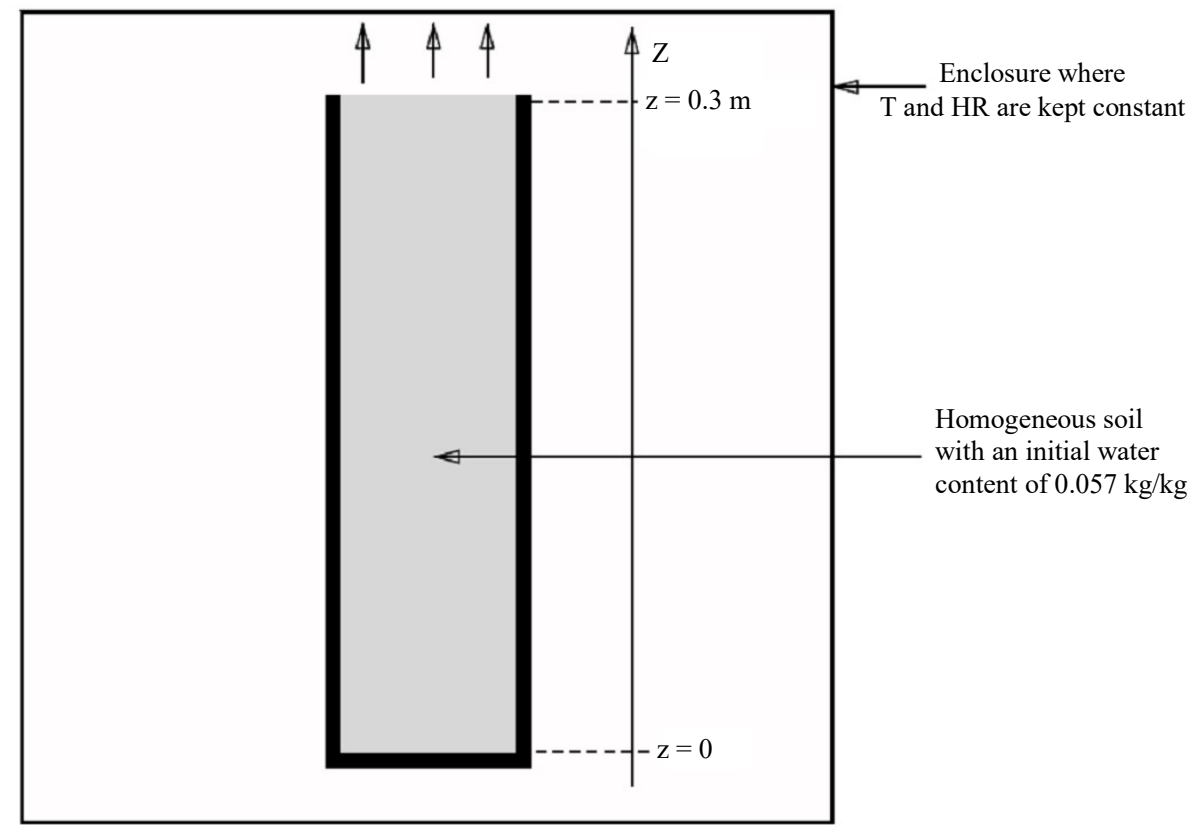

Fig. 4: Schematization of the physical model

The phase change coefficient, $L$, introduced in this relation, should depends on the state variables such as the water content, the temperature and on the nature of the soil.

\section{Physical Model}

The physical configuration of the medium that we consider in this study is represented in Fig. 4. It is a column of soil compacted with uniform water content and subjected to drying conditions from the top face. The soil column is closed on the lower base and on the side parts so as to impose a unidirectional transfer thus approaching the natural operating conditions of a surface layer of a soil. The upper part is subjected to drying under a constant temperature of $30^{\circ} \mathrm{C}$ and an atmosphere of constant relative humidity of $30 \%$. The physical phenomena considered are therefore: Filtration of liquid water, diffusion of water vapour and liquid-vapour phase change.

This configuration is perfectly identical to the experimental studies conducted by (Ouedraogo et al., 2013), this to allow the comparison of the results for the study of the non-equilibrium in the transfer at low water contents.

\section{Initial Conditions}

- We consider a soil with uniform initial water content: $w_{i}=0.057 \mathrm{~kg} \cdot \mathrm{kg}^{-1}$.

- Under these conditions, we admit that the water vapour pressure, in the initial state, is equal to the equilibrium vapour pressure of water at temperature $T$. This means that at the initial time, on the whole column of soil:

$$
p_{\text {veq }}=p_{\text {veq }}=a_{w}(w) p_{v s}(T)
$$

\section{Boundary Conditions}

- At side $z=0$, the liquid water flux $\Phi_{e}$ and vapour flux $\Phi_{v}$ are zero:

$$
\left.\Phi_{e}\right|_{z=0}=\left.\Phi_{v}\right|_{z=0}=0
$$

- At $z=h$, the partial pressure of the water vapour is set by the relative humidity, HR of the ambient air:

$$
p_{v}=H R \times p_{v s}(T)
$$

\section{Numerical Scheme}

The equations are discretized based on a finitevolume formulation with a one-dimensional regular mesh where the unknown, $w$, is at the centre of the grid blocks. A first order upstream scheme is used to describe the convective term. Temporal discretization is carried out by a fully implicit scheme to obtain unconditional numerical stability. To handle non-linearities, the Newton-Raphson method ensures accurate convergence with a moderate time-step.

\section{Results and Discussion}

\section{Phase Change: Experimentation and Modeling}

The phase change rate is determined experimentally in the laboratory by the method described in (Lozano et al., 
2008) then modeled before being integrated into the simulation code of the coupled transfers of liquid water and water vapour. Different models have been proposed in the literature to represent the rate of change of liquid/vapour phase in a porous material (Lozano et al., 2008; Trautz et al., 2015; Li et al., 2019). In this study, we opted for the model in (Lozano et al., 2008) which has been widely used in similar studies (Chammari et al., 2008; Benet et al., 2009; Ouedraogo et al., 2013). The complete model of the phase change coefficient proposed as functions of the water content and the vapour partial pressure is characterized by 3 coefficients $\left(L_{e q}, k, r\right)$ through the following expressions:

- $\quad$ Close to equilibrium, i.e., $r \leq \frac{p_{v}}{p_{v e q}} \leq 1$ :

$$
L=L_{e q}
$$

- $\quad$ Far from equilibrium, i.e., $0 \leq \frac{p_{v}}{p_{v e q}} \leq r$ :

$$
L=L_{e q}+k\left(r-\frac{p_{v}}{p_{v e q}}\right)
$$

The neighbourhood of an equilibrium situation, i.e., when the vapour partial pressure is close to its equilibrium value, corresponds to the validity domain of the linear thermodynamics of irreversible processes and a constant phenomenological coefficient is observed (Ouedraogo et al., 2013). Outside of this domain, i.e., far from equilibrium, an affine dependence on the vapour partial pressure is obtained and the phase change rate is highly increased. Concerning the soil under investigation, the influence of the water content on model parameters $\left(L_{e q}, r\right)$ is presented in Fig. 5 and 6 while the third parameter $r$ has been found to be fairly constant, $r=0.98$.

Some bell-shaped curves are generally observed, where the maximum around $w=0.035 \mathrm{~kg} \cdot \mathrm{kg}^{-1}$ is roughly the upper limit of the hygroscopic domain. Above this maximum, the phase change rate decreases since the liquid-gas interface area reduces. For water content greater than $6 \%$, the gas phase is occluded and phase change cannot be activated. Below the maximum, when hygroscopic effects become predominant, the intensity of solid-liquid interactions increase in the adsorbed layers. The supplementary energy required for water desorption decreases the phase change rate leading to lower values of the coefficient.

The Fig. 7 represents the slope $k$ as a function of the water content.

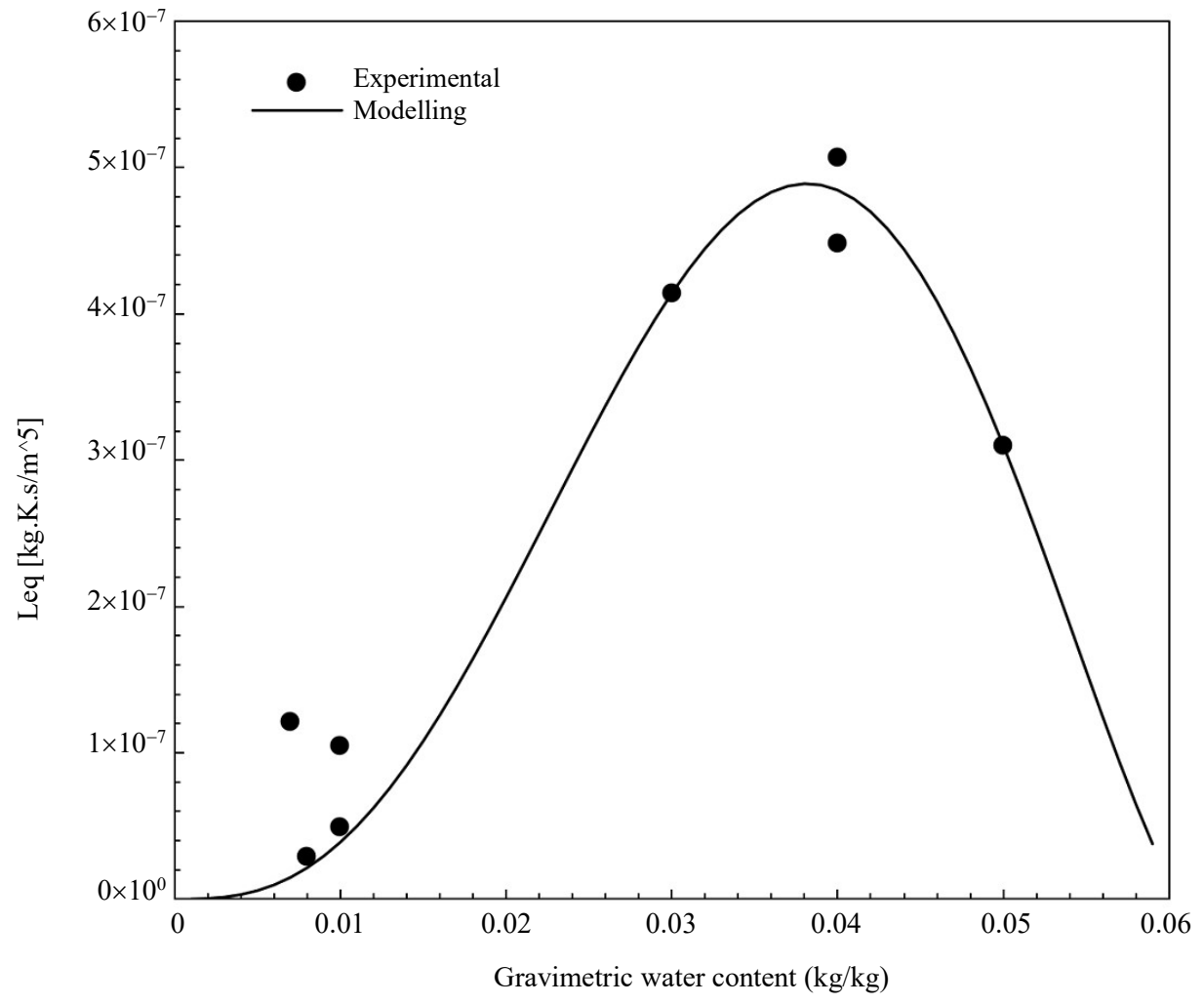

Fig. 5: Evolution of the equilibrium phase change coefficient $L_{e q}$ and its modeling as a function of the water content 


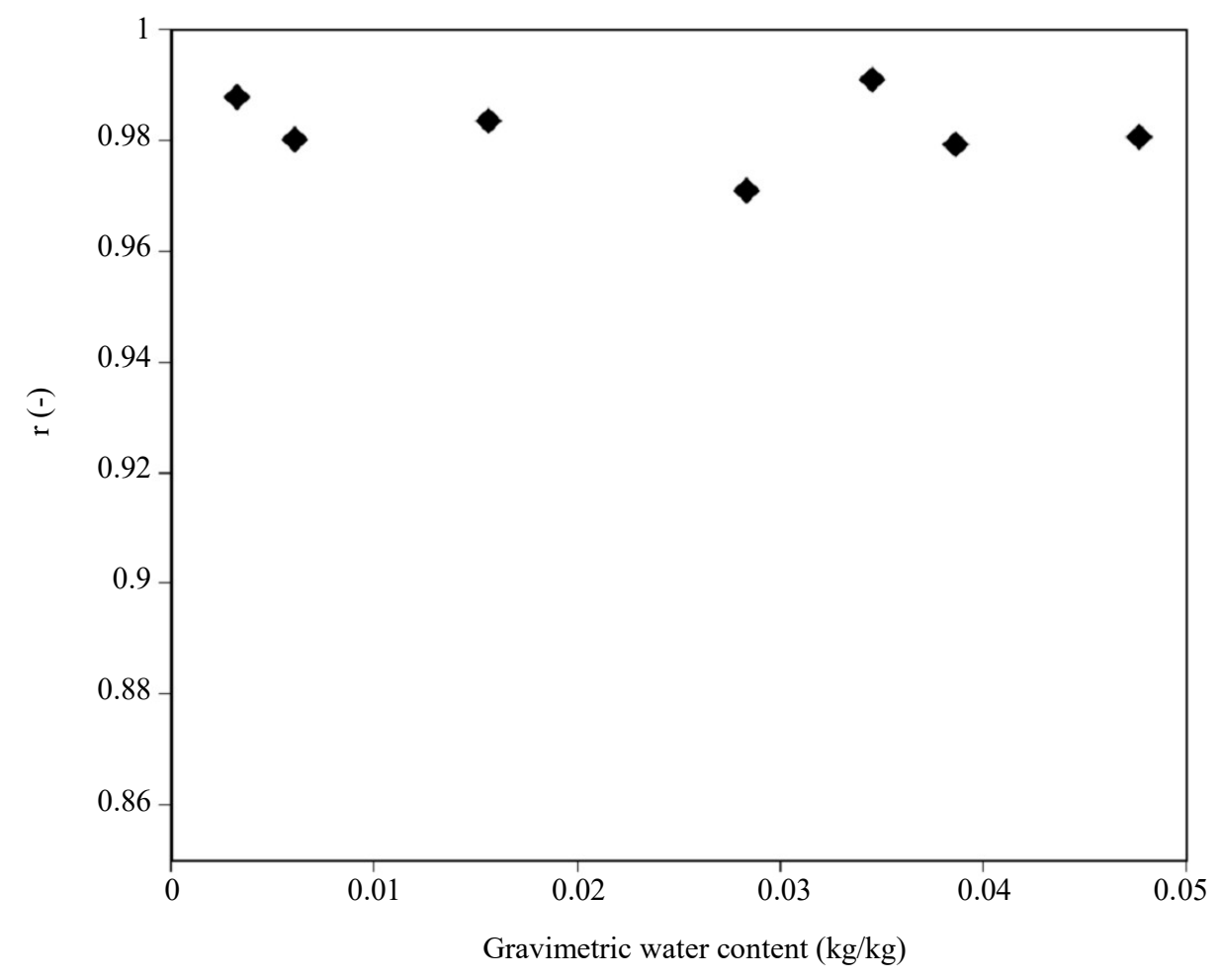

Fig. 6: Variation of the ratio $r$ as a function of the water content

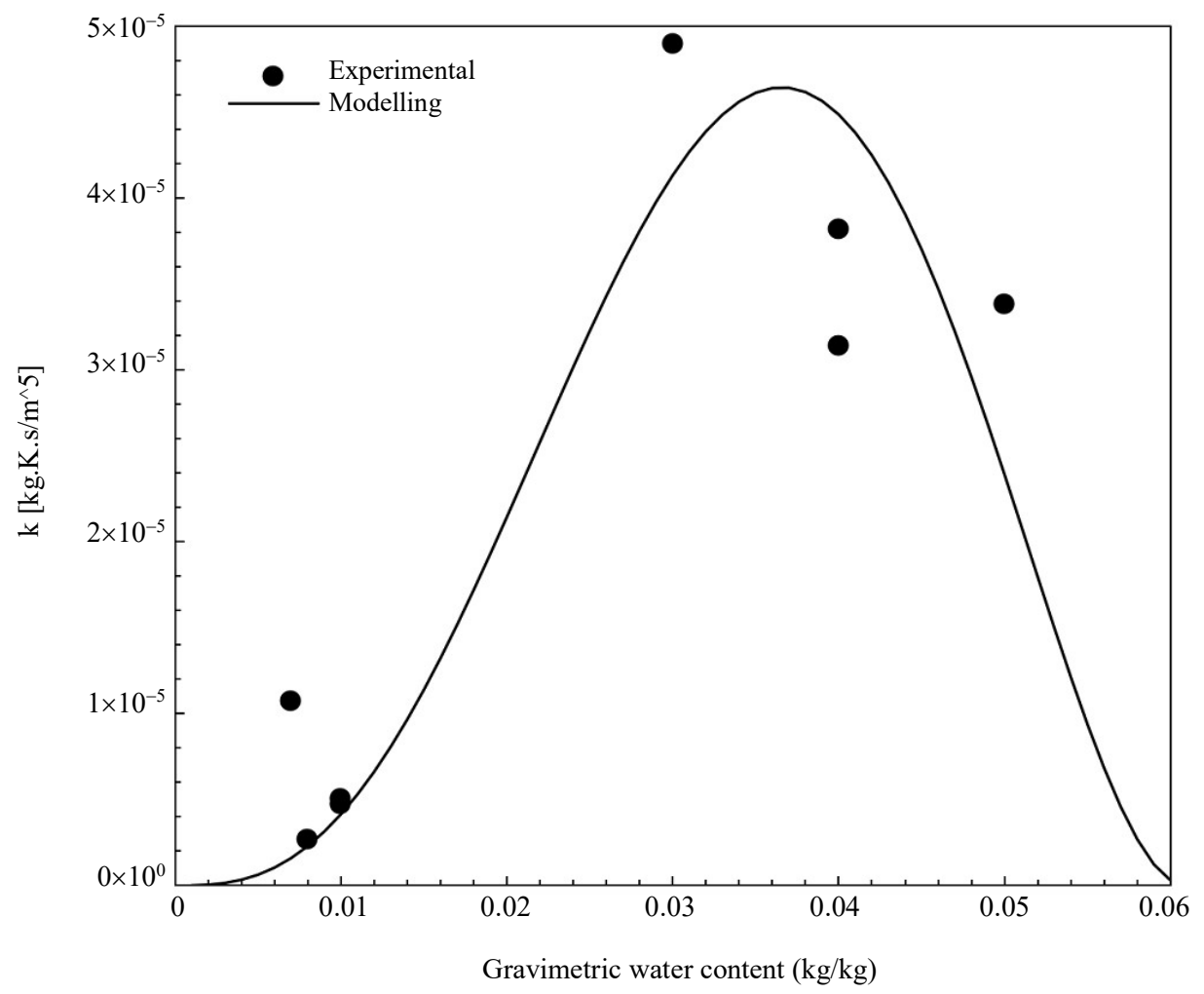

Fig. 7: Variation of the slope $k$ as a function of the water content 
The modeled expressions for $L_{e q}$ and the slope $k$ as a function of the water content are as follows:

$$
\begin{aligned}
& L_{e q}(w)=(w-0.0606)\left(-0.9019 w^{3}+13.3688 w^{4}\right) \\
& k(w)=(w-0.0609)\left(-97.48 .1 w^{3}+1599.7720 w^{4}\right)
\end{aligned}
$$

\section{Phase Change Flux}

We use the unidirectional model exposed in the above sections to simulate water transfers in soil at low water contents by filtration of liquid water and diffusion of water vapour coupled by liquid/gas phase change. This study is a numerical case but in the same configuration as the experimental and numerical studies carried out by (Ouedraogo et al., 2013) for a soil with characteristics very close to NH1.

The phase change phenomenon is not really quantified by a flux term $\left[\mathrm{kg} \cdot \mathrm{m}^{-2} \cdot \mathrm{s}^{-1}\right]$ but by a volumetric rate $\left[\mathrm{kg} \cdot \mathrm{m}^{-3} \cdot \mathrm{s}^{-1}\right]$. Thus, we will make a qualitative comparison. We consider the relation (Eq. 18) to represent the nonequilibrium phase change flux in terms of surface flux by multiplying the rate $\hat{\rho}$ by a length. We choose $d z$ which represents the discretization space step used in the numerical simulation:

$$
\hat{J}_{v}=\hat{\rho} d z
$$

The simulation is carried out by considering two model of the un relative permeability function $(K r)$ :

- The capillary model of Van Genuchten (1980) Mualem (1976) (Eq. 3) that we denoted in the figures below by " $K r-V G 80-M$ ", the same model in (Ouedraogo et al., 2013)

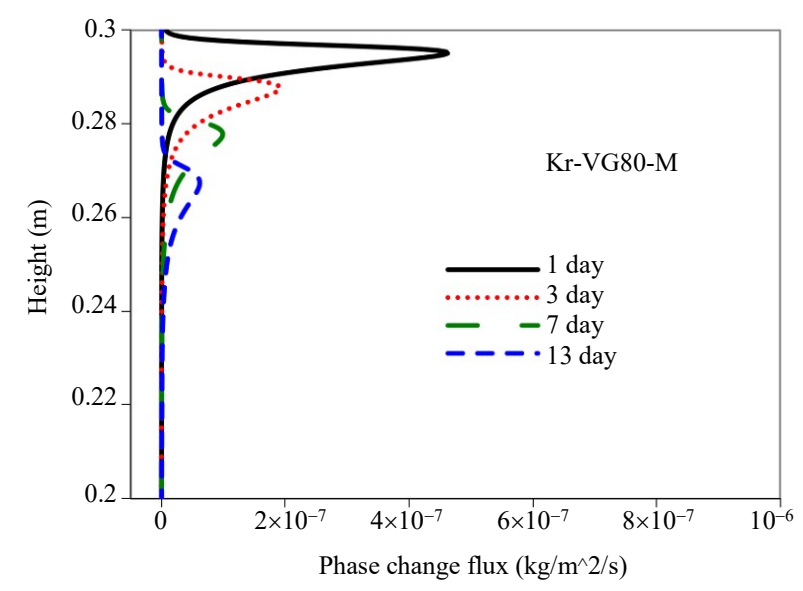

(a)
- $\quad$ The model considering the film flow (Eq. 4) that we denoted in the figures below by " $K r-K 17$ ".

The simulated profiles of the phase change flux, at different times, are shown in the Fig. 8a with the (Van Genuchten, 1980; Mualem, 1976) model and in the Fig. $8 \mathrm{~b}$ with the model considering the film flows.

The non-equilibrium phase change flux is remarkable in the Fig. 8a with an abrupt change in its value in an area of thickness about $5 \mathrm{~cm}$ from the soil-atmosphere interface. This variation is not observed in the Fig. 8b. Thus, it can be noted that considering the film flow mode profoundly modifies the evolution of the phase change flux and brings the area subjected to strong variations in flux very close to the surface exposed to the atmosphere. Does this observation call into question the rejection formulated in (Ouedraogo et al., 2013) for the local equilibrium assumption made in most case of water (liquid and vapour) transfer in the porous media? This hypothesis specifies that, on each point of the soil profile, the liquid and gas phases are in equilibrium. In our case, it implies that at all times, the effective pressure of the water vapour $p_{v}$ is equal to the equilibrium vapour pressure $p_{v e q}$. In other words, the vapour pressure is maintained at its equilibrium value. Let us study more finely the rapport between these two quantities which expresses the non-equilibrium.

\section{Assessment of the Non-Equilibrium}

To assess the liquid/gas non-equilibrium, the ratio of the effective pressure of the vapour divided by its equilibrium value is proposed:

$$
\theta=\frac{p_{v}}{p_{v e q}}
$$

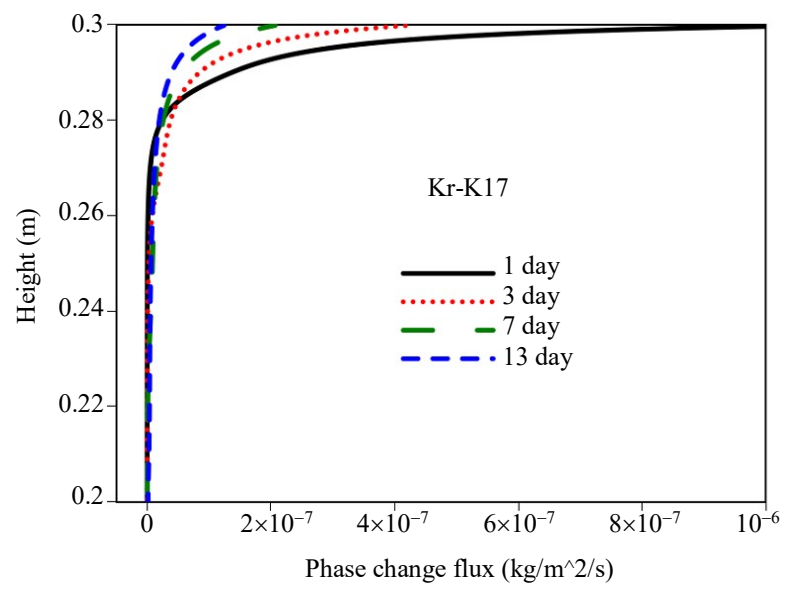

(b)

Fig. 8: Phase change flux simulated with: (a) The model of Van Genuchten (1980) -Mualem (VGM80-M) and (b) the model K17 considering the film flows 


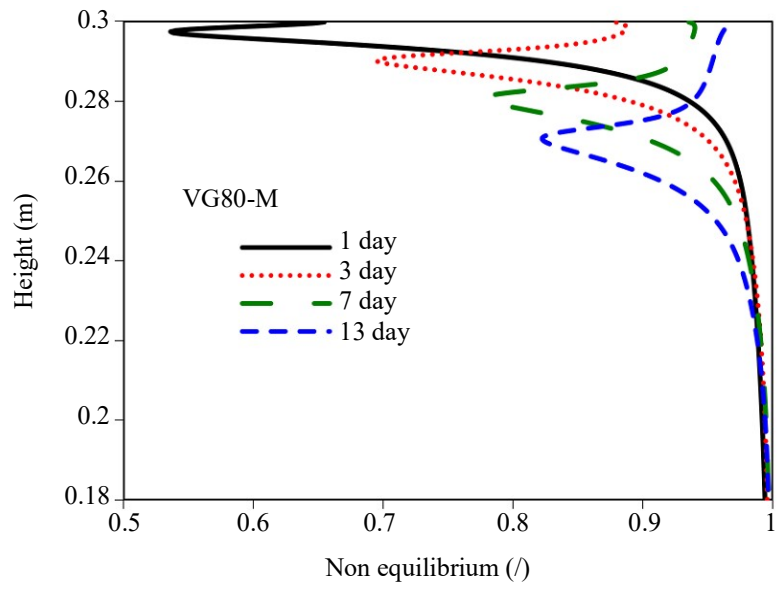

(a)

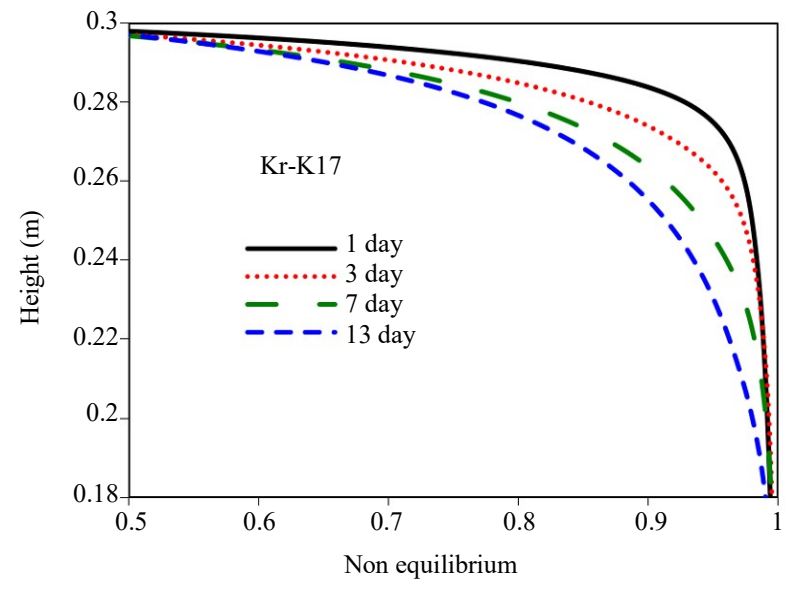

(b)

Fig. 9: Non-equilibrium profiles simulated with: (a) the model of Van Genuchten (1980) -Mualem (VGM80 - M) and (b) the model K17 considering the film flows

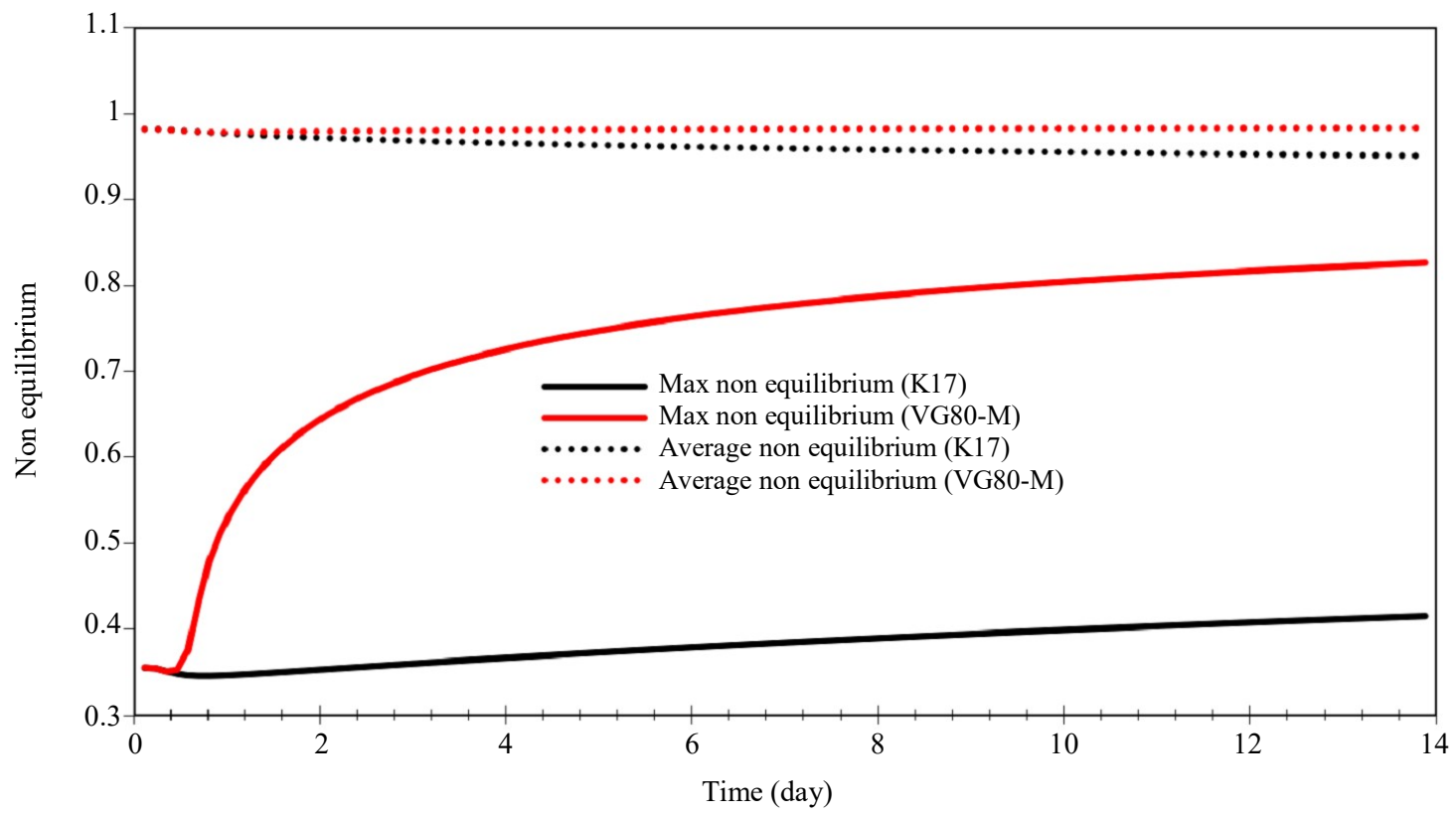

Fig. 10: Comparison of the evolutions of the average and maximum non-equilibrium for the two models of the relative permeability considered

This criterion is plotted along the soil column in Fig. 9a for (Van Genuchten, 1980; Mualem, 1976) capillary model for the relative permeability function and the Fig. $9 \mathrm{~b}$ with the model considering the film flows. The profiles in the Fig. 9a have the same shape as those from (Ouedraogo et al., 2013) but a form completely different from the case where we consider the film flows (Fig. 9b) in this water state of the soil. By comparing the two figures (Fig. 9a and 9b), one can note that the vapour pressure can differ greatly from its equilibrium value in the case of capillary flow only and moderately when the film flow has been taken account.
We also plot in the Fig. 10 the temporal evolutions of the average and maximum non-equilibrium for the two models of the relative permeability function. With the Van Genuchten (1980) - Mualem (1976) capillary model, the sudden growth of maximum non-equilibrium, for a short time $(t<4$ days) corresponds to the establishment of the vapour profile due to diffusion phenomena. This transient behavior is not observed in the case where the model considers both capillary and film flows.

Considering the film flow, the filtration of the liquid phase of the water is extended up to the hygroscopic state of the soil. By comparing the two modeling 
approaches of liquid filtration phenomena, considering film flows has the consequence to maintain a significant flux of liquid phase into the soil surface for longer times. Figure 11 where we plotted the profiles of liquid water flux along the soil column for each model of relative permeability clearly illustrate this situation. We note on each of the Fig. 11a and 11b, two zones: A first zone, over an altitude of $25 \mathrm{~cm}$, where the liquid phase flux increase to reach a maximum and a second zone, between 25 and $30 \mathrm{~cm}$ where the liquid phase flux decrease to reach zero at the interface with the atmosphere. By comparing the Fig. 11a and 11b, we can remember that do not consider film flows in low water content range does not only underestimate the flux but the depth of the area where the flux is important: About $10 \mathrm{~cm}$ deep with the capillary model by Van Genuchten (1980) - Mualem (1976) and $15 \mathrm{~cm}$ for the model extended to film flow.

The unexpected behavior of the non-equilibrium in the transfers of the liquid and gas phases in the soil in the case where we consider the film flows can be explained by a predominance of the movement of the water adsorbed on the solid matrix in liquid form. Thus, this phenomenon occurs to the detriment of the diffusion of the vapour phase which follows the initiation of the process of liquid/vapour phase change in the soil subjected to our controlled atmosphere.

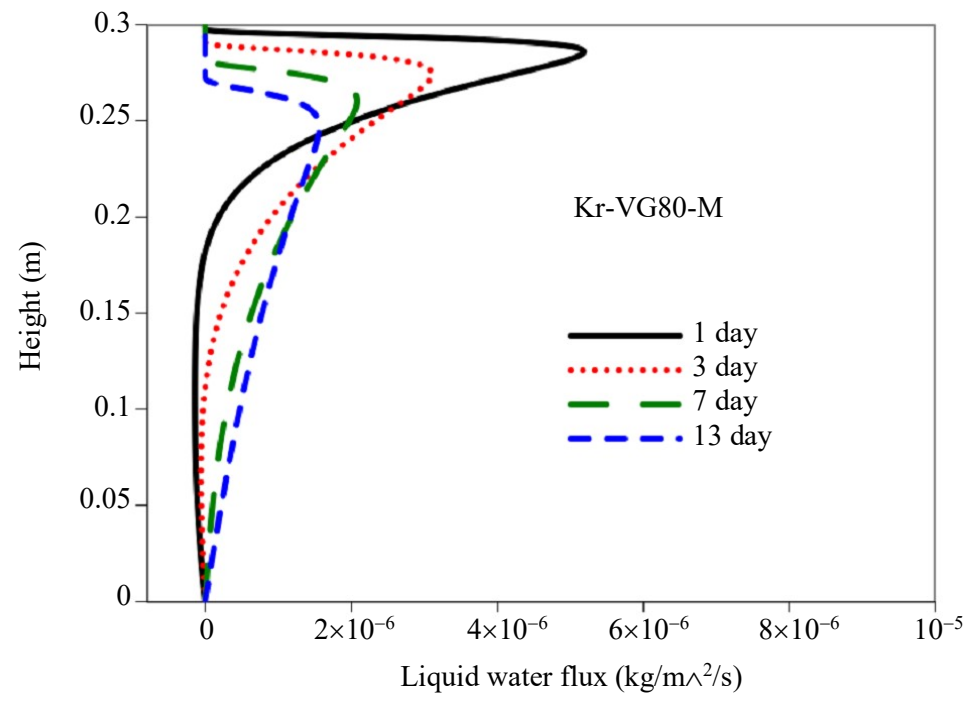

(a)

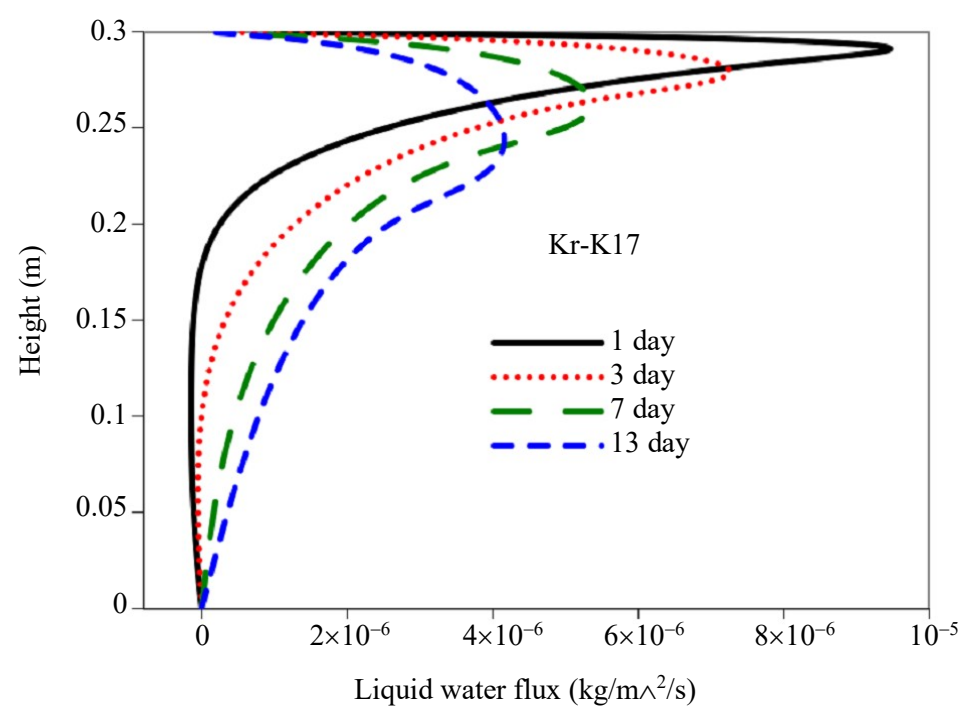

(b)

Fig. 11: Liquid water flux simulated with: (a) the model of Van Genuchten (1980) -Mualem (VGM80 - M) and (b) the model K17 considering the film flows 


\section{Conclusion}

In this study, we have presented an experimental characterization of the non-equilibrium phase change for NH1, a surface layer of Nasso soil. The near and fartoequilibrium phase change coefficients modeled from the experimental data, supplemented with the hydrodynamic functions described were used to feed a numerical program to simulate liquid and vapour water transfers. The physical environment is likened to a column of soil, the upper end of which is exposed to an atmosphere regulated in temperature and relative humidity and therefore subject to evaporative phenomena.

We noted that the shapes of the profiles of fluxes (liquid water, liquid/vapour non-equilibrium phase change) simulated using the capillary model (model of Van Genuchten (1980) - Mualem (1976)) are very different from those obtained with the model considering the film flow (model in Kébré et al., 2017). In this last case, the liquid/gas non-equilibrium is not noticeable as in the case of the capillary model. It seems that the film flows occult the water vapour diffusion by delaying the phase change process, therefore a lower concentration of water vapour into the soil than in the case where the capillary flow alone has been considered.

What are the physical and fundamental limits for the observation of film flows when the medium is in an arid environment? Thus, to better understands the observed behavior in thermodynamic non-equilibrium with the global dynamics of liquid water or vapour in the soil, a series of experiments will be necessary. In addition, the mechanism of the vapor transport in soil under nonequilibrium and equilibrium conditions should be more investigated following pioneers works of (Smits et al., 2011; Li et al., 2019; Seredyński et al., 2020; etc.).

\section{Acknowledgement}

We pay tribute through this publication to Prof. JeanClaude Bénet from the Laboratory of Mechanics and Civil Engineering (LMGC) of Montpellier, (now deceased), for his contribution to the realization of this work.

\section{Author's Contributions}

Marcel Bawindsom Kébré, François Ouédraogo and Fabien Cherblanc: Designed the research plan, participated in all programming and calculation, coordinated the data analysis and contributed to the writing of the paper.

Bétaboalé Naon and François Zougmoré: Contributed to methodology and discussions on results.

\section{Ethics}

This article is original and contains unpublished material. The corresponding author confirms that the other authors have read and approved the manuscript and that no ethical issues are involved.

\section{References}

Aljerf, L. (2018). High-efficiency extraction of bromocresol purple dye and heavy metals as chromium from industrial effluent by adsorption onto a modified surface of zeolite: kinetics and equilibrium study. Journal of Environmental Management, 225, 120-132. https://doi.org/10.1016/j.jenvman.2018.07.048

Benet, J. C., Lozano, A. L., Cherblanc, F., \& Cousin, B. (2009). Phase change of water in a hygroscopic porous medium. Phenomenological relation and experimental analysis for water in soil. Journal of Non-Equilibrium Thermodynamics, 34(2), 133-153. https://doi.org/10.1515/JNETDY.2009.008

Burdine, N. (1953). Relative permeability calculations from pore size distribution data. Journal of Petroleum Technology, 5(03), 71-78. https://doi.org/10.2118/225-G

Chammari, A., Naon, B., Cherblanc, F., Cousin, B., \& Bénet, J. C. (2008). Interpreting the drying kinetics of a soil using a macroscopic thermodynamic nonequilibrium of water between the liquid and vapor phase. Drying Technology, 26(7), 836-843. https://doi.org/10.1080/07373930802135998

Campbell, G. S. (1985). Soil physics with BASIC: transport models for soil-plant systems. New York: Elsevier. ISBN-10: 0080869823.

Fredlund, D. G., \& Xing, A. (1994). Equations for the soil-water characteristic curve. Canadian Geotechnical Journal, 31(4), 521-532. https://doi.org/10.1139/t94-061

Halder, A., Dhall, A., \& Datta, A. K. (2011). Modeling transport in porous media with phase change: applications to food processing. Journal of Heat Transfer, 133(3). https://doi.org/10.1115/1.4002463

Khaleel, R., Relyea, J. F., \& Conca, J. L. (1995). Evaluation of van Genuchten-Mualem relationships to estimate unsaturated hydraulic conductivity at low water contents. Water Resources Research, 31(11), 2659-2668. https://doi.org/10.1029/95WR02309

Kébré, M. B., Cherblanc, F., Ouedraogo, F., Jamin, F., Naon, B., Zougmoré, F., \& Benet, J. C. (2017). Water flow in soil at small water contents: a simple approach to estimate the relative hydraulic conductivity in sandy soil. European Journal of Soil Science, 68(2), 167-176. https://doi.org/10.1111/ejss.12408

Kébré, M. B., Ouedraogo, F., Betaboalé, N., Zougmoré, F., \& Cherblanc, F. (2013). Soil-Water Characteristic Curve Modeling at Low Water Content: Empirical and Semi-Empirical Approaches. International Journal of Engineering Research, 2(7), 432-437. https://hal.archives-ouvertes.fr/hal-00948643/ 
Kébré, M. B. (2013). Gestion des Ressources en Eau dans les Régions Arides: Analyse Expérimentale d'un Sol Type du Burkina Faso et Modélisation Numérique des Transferts d'Eau (Doctoral dissertation, Université Montpellier II-Sciences et Techniques du Languedoc). https://tel.archivesouvertes.fr/tel-01012040/

Levitt, D. G., \& Young, M. H. (2007). Soils: Hygroscopic Water Content. In: Trimble, S. W., (Ed.), Encyclopedia of Water Science, (pp.1136-1139), Taylor \& Francis, ISBN $0849396190,9780849396199$.

Li, Z., Vanderborght, J., \& Smits, K. M. (2019). Evaluation of model concepts to describe water transport in shallow subsurface soil and across the soil-air interface. Transport in Porous Media, 128(3), 945-976. https://doi.org/10.1007/s11242-018-1144-9

Lozano, A. L., Cherblanc, F., Cousin, B., \& Bénet, J. C. (2008). Experimental study and modelling of the water phase change kinetics in soils. European Journal of Soil Science, 59(5), 939-949. https://doi.org/10.1111/j.1365-2389.2008.01050.x

Massman, W. J. (2015). A non-equilibrium model for soil heating and moisture transport during extreme surface heating: the soil (heat-moisture-vapor) HMV-Model Version 1. Geoscientific Model Development, $\quad 8(11), \quad 3659-3680$. https://doi.org/10.5194/gmd-8-3659-2015

Millington, R. J., \& Quirk, J. P. (1961). Permeability of porous solids. Transactions of the Faraday Society, 57 , 1200-1207. https://pubs.rsc.org/en/content/articlelanding/1961/tf /tf9615701200/unauth\#!divAbstract

Mitarai, N., \& Nori, F. (2006). Wet granular materials. Advances in Physics, 55(1-2), 1-45. https://doi.org/10.1080/00018730600626065

Moldrup, P., Olesen, T., Gamst, J., Schjønning, P., Yamaguchi, T., \& Rolston, D. E. (2000). Predicting the gas diffusion coefficient in repacked soil water-induced linear reduction model. Soil Science Society of America Journal, 64(5), 1588-1594. https://doi.org/10.2136/sssaj2000.6451588x

Mualem, Y. (1976). A new model for predicting the hydraulic conductivity of unsaturated porous media. Water Resources Research, 12(3), 513-522. https://doi.org/10.1029/WR012i003p00513
Novak, M. D. (2019). Validity of assuming equilibrium between liquid water and vapor for simulating evaporation. Water Resources Research, 55(11), 9858-9872. https://doi.org/10.1029/2019WR025113

Nuske, P., Joekar-Niasar, V., \& Helmig, R. (2014). Nonequilibrium in multiphase multicomponent flow in porous media: An evaporation example. International Journal of Heat and Mass Transfer, 74, 128-142. https://doi.org/10.1016/j.ijheatmasstransfer.2014. 03.011

Ouedraogo, F., Cherblanc, F., Naon, B., \& Bénet, J. C. (2013). Water transfer in soil at low water content. Is the local equilibrium assumption still appropriate?. Journal of Hydrology, 492, 117-127. https://doi.org/10.1016/j.jhydrol.2013.04.004

Seredyński, M., Wasik, M., Łapka, P., Furmański, P., Cieślikiewicz, Ł., Pietrak, K., ... \& Jaworski, M. (2020). Analysis of Non-Equilibrium and Equilibrium Models of Heat and Moisture Transfer in a Wet Porous Building Material. Energies, 13(1), 214. https://doi.org/10.3390/en13010214

Smits, K. M., Cihan, A., Sakaki, T., \& Illangasekare, T. H. (2011). Evaporation from soils under thermal boundary conditions: Experimental and modeling investigation to compare equilibrium-and nonequilibrium-based approaches. Water Resources Research, 47(5). https://doi.org/10.1029/2010WR009533

Tamizdoust, M. M., \& Ghasemi-Fare, O. (2020). Utilization of Nonequilibrium Phase Change Approach to Analyze the Nonisothermal Multiphase Flow in Shallow Subsurface Soils. Water Resources Research, 56(10), e2020WR027381. https://doi.org/10.1029/2020WR027381

Trautz, A. C., Smits, K. M., \& Cihan, A. (2015). Continuum-scale investigation of evaporation from bare soil under different boundary and initial conditions: An evaluation of nonequilibrium phase change. Water Resources Research, 51(9), 7630-7648. https://doi.org/10.1002/2014WR016504

Van Genuchten, M. T. (1980). A closed-form equation for predicting the hydraulic conductivity of unsaturated soils. Soil Science Society of America Journal, 44(5), 892-898. https://doi.org/10.2136/sssaj1980.036159950044000 $50002 x$ 